\title{
Room temperature optical thermometry based on the luminescence of the SiV defects in diamond
}

\author{
Caius Miller ${ }^{1}$, Laurits Puust $^{1}$, Valter Kiisk ${ }^{1}$, Evgeny Ekimov ${ }^{2}$, Igor Vlasov ${ }^{3}$, Yurii \\ Orlovskii ${ }^{1,3}$, and Ilmo Sildos ${ }^{1, *}$ \\ ${ }^{1}$ Institute of Physics, University of Tartu, W. Ostwaldi Str. 1, EE50411 Tartu, Estonia \\ ${ }^{2}$ Institute for High Pressure Physics RAS, Kaluzhskoe Road 14, Troitsk, Moscow 108840, Russia \\ ${ }^{3}$ Prokhorov General Physics Institute RAS, Vavilov Street 38, Moscow 119991, Russia
}

\begin{abstract}
SiV-containing microcrystals of diamond are synthesised by using high-pressure high-temperature treatment of a mixture of pertinent organic-inorganic precursors. Photoluminescence of $\mathrm{SiV}$ defects were investigated with the aim to use the microcrystals for optical temperature sensing in near infrared at room temperature based on temperaturedependent shift of the $740 \mathrm{~nm}$ zero-phonon line of $\mathrm{SiV}$ photoemission.
\end{abstract}

\section{Introduction}

Biomedical applications require nontoxic photoresistant optical markers absorbing and emitting in the NIR biological window of biotissues. Several impurities in diamond, such as $\mathrm{SiV}$ and NE8 defect centres with spectrally narrow zero-phonon lines (even at room temperature), satisfy these demands $[1,2]$.

\section{Experimental}

Diamond microcrystals (typical size $\sim 1 \mu \mathrm{m}$ ) containing $\mathrm{SiV}$ defects were synthesized from a mixture of adamantane $\left(\mathrm{C}_{10} \mathrm{H}_{16}\right)$ and tetraphenylsilane $\left(\mathrm{C}_{24} \mathrm{H}_{20} \mathrm{Si}\right)$ in a ratio of $\mathrm{Si} / \mathrm{C}$ $0.28 \%$. An apparatus of uniaxial compression and the high-pressure chamber of "Toroid15 " type were used for the synthesis [3]. The diamond sample was synthesized in titanium capsule at a pressure of about $9 \mathrm{GPa}$ and a temperature of $1600{ }^{\circ} \mathrm{C}$, the exposure time at the synthesis temperature was 10 seconds.

Under $488 \mathrm{~nm}$ laser excitation the $\mathrm{SiV}$ defects possessed an intense emission spectrum with a quite sharp zero-phonon line (ZPL) at $740 \mathrm{~nm}$ over a wide temperature range (Fig. 1). The spectral position of the ZPL shows a strong red-shift by increase of temperature (Fig. 2). Acceptable fitting could not be achieved by the conventional $T^{4}$ dependence but required additional $T^{2}$ term to take into account softening of bonds in the excited electronic state in accordance of a modified model of the ZPL of impurity centers in solids [4].

\footnotetext{
*Corresponding author: ilmo.sildos@ut.ee
} 


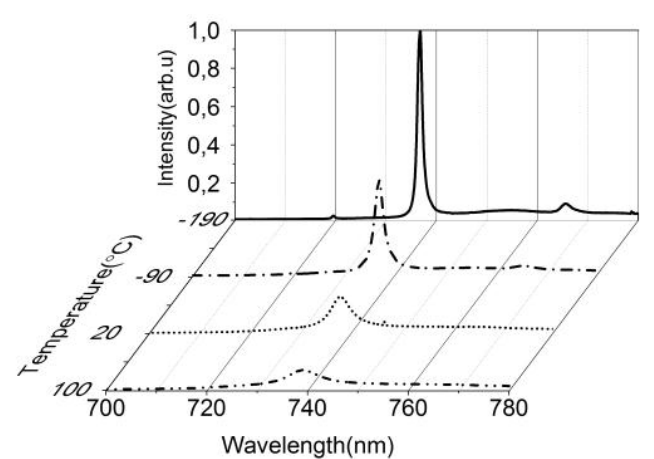

Fig. 1. Temperature dependence of $\mathrm{SiV}$ emission in a diamond microcrystal. Excitation with $488 \mathrm{~nm} \mathrm{Ar}^{+}$ laser line

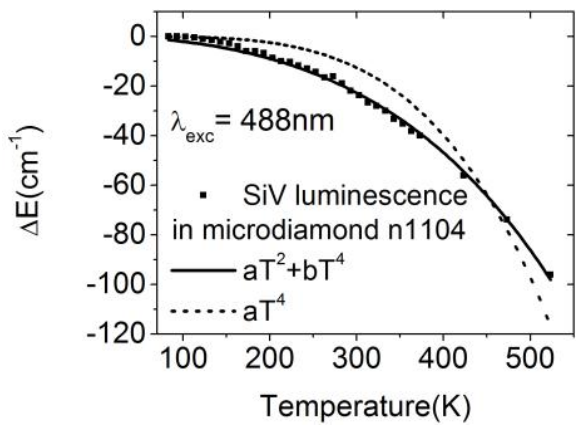

Fig. 2. Thermally induced shift of the $740 \mathrm{~nm}$ $\mathrm{ZPL}$ in the emission spectrum of $\mathrm{SiV}$ defects in diamond micro-crystals.

\section{Concluding remarks}

A narrow $740 \mathrm{~nm}$ ZPL and its strong position dependence on temperature are promising for a sensitive $(\sim 1 \mathrm{~K})$ optical thermometry around RT. Preparation of SiV-containing nanodiamonds by using the above described HPHT technique is in progress. We further intend to test the thermo-sensing capabilities of the nanoparticles in aqueous media and implement imaging and thermometry with different cell cultures.

The research of Estonian and Russian authors was supported by EU through the ERA.Net Rusplus project "88 DIABASE" and by Russian Science Foundation (grant No 14-12-01329), respectively.

\section{References}

1. Ch. T. Nguyen, R. E. Evans, A. Sipahigil, M. K. Bhaskar, D. D. Sukachev, V. N. Agafonov, V. A. Davydov, L. F. Kulikova, F. Jelezko, M. D. Lukin, arXiv:1708.05419v1 (2017)

2. I. Sildos, A. Loot, V. Kiisk, L. Puust, V. Hizhnyakov, A. Yelisseyev, A. Osvet, I. Vlasov, Diamond and Related Materials, 76, 27 (2017)

3. E. A. Ekimov, O. S. Kudryavtsev, N. E. Mordvinova, O. I. Lebedev, and I. I. Vlasov ChemNanoMat, 4, 269 (2018)

4. V. Hizhnyakov, J. Phys. Cond. Matt., 24, 104011 (2012) 